Research Articles

\title{
Combination of Serological Tests (Anti-CCP Antibody, Rheumatoid Factor IgM ELISA and Latex Test) are more Useful in Detection of Rheumatoid Arthritis
}

\author{
${ }^{1}$ Anindya Das, ${ }^{1}$ Chimanjita Phukan and ${ }^{2}$ Chitralekha Baruah \\ ${ }^{1}$ Department of Microbiology, Gauhati Medical College and Hospital, Guwahati, India \\ ${ }^{2}$ Department of Medicine, Gauhati Medical College and Hospital, Guwahati, India
}

\author{
Article history \\ Received: $15-11-2016$ \\ Revised: 27-01-2017 \\ Accepted: 18-09-2017 \\ Corresponding Author: \\ Anindya Das, \\ Department of Microbiology, \\ Gauhati Medical College and \\ Hospital, Guwahati, India \\ Email: dranindyadas20@gmail.com
}

\begin{abstract}
Rheumatoid arthritis is a polyarticular and chronic inflammatory disease occurring throughout the world. To prevent significant joint damage, early diagnosis and proper treatment is of paramount importance. Though patients are diagnosed clinically supported by radiography and serological tests, early disease may present with non-specific arthritis and absence of specific radiographic findings. Though anti-CCP antibody is used for the diagnosis and may be found in early disease, recently some variability of results has been observed in some studies. In this context present study was carried out to combine anti-CCP antibody, rheumatoid factor IgM ELISA and Latex agglutination test to observe the combined specificity and sensitivity of the tests and the tests were compared with each other to examine the correlation between them.
\end{abstract}

Keywords: Anti-CCP Antibody, Rheumatoid Factor, Latex Agglutination Test, Combined Specificity, Resultant Sensitivity, ELISA

\section{Introduction}

Rheumatoid Arthritis (RA) may produce in many cases a significant level of morbidity (Arnett et al., 1988). Though the diagnosis of rheumatoid arthritis is primarily clinical, based on signs and symptoms of chronic inflammatory arthritis with laboratory radiographic results providing important supplemental information, in many patients early disease presents with non-specific arthritis. Recently there is significant motivation among clinicians to diagnose rheumatoid arthritis patients, early in the course of the disease, because the recently developed disease modifying drugs and biological agents are notably helpful in long term outcomes of the patients (O'Dell, 2003). However, the overenthusiastic initiation of treatment with disease modifying agents, without diagnosing RA accurately can cause a lot of harm to the patient. It is also very important to identify the patients who will have progressive, erosive disease, as early aggressive treatment may help them most (Lee and Schur, 2003). Though radiography is the most common imaging modality in RA patients and allows easy serial comparison for assessment of disease progression, the main disadvantage is the absence of specific radiographic findings in early disease, since visualization of erosions may only be seen later (Guermazi et al., 2004).
The first autoantibody in RA, Rheumatoid Factor (RF), was described by Dr Waaler (1940). IgM, IgG and IgA isotypes of RF occur in sera from patients with RA, although the IgM isotype is the one, most frequently measured by commercial laboratories. The IgM or IgG RFs in the joints activate complement and recruit macrophages, neutrophils and lymphocytes and induce inflammation (Soltys and Axford, 1997). However, RF has been observed in many other autoimmune diseases, infectious diseases and even in healthy people. So, not all rheumatoid factors cause disease and many studies are recently going on to characterize the difference between "pathological" and "physiological" RFs (Brian et al., 1998).

Nienhuis and Mandema (1964) described a specific antibody for rheumatoid arthritis called Antiperinuclear Factor (APF) as these antibodies combine with the components of the keratohyaline granules which are located close to the nucleus of buccal mucosa cells of adult people. Another group of antibodies found in RA patients, named Antikeratin Antibodies (AKA), which bind to keratin-like structures of stratum corneum of epidermis, was mentioned by Young (1979). Sebbag et al. (1995) showed that antiperineuclear factors and antikeratin antibodies combine with the same antigen identified as filaggrin (filament aggregating protein). 
Schellekens et al. (1998) documented that, citrullination of filaggrin plays an important role in autoantigenic property. APF and AKA mainly target the citrullinated filaggrin antigen. Based on that finding, Schellenkens GA first prepared an enzyme immunoassay, using human filaggrin as substrate (Schellekens et al., 1998). Through several stages of development the assay was later improved as second generation anti-CCP test with a sensitivity of 70$80 \%$ and specificity of $95-98 \%$ in established rheumatoid arthritis (van Venrooij et al., 2011).

Citrullination occurs normally inside the damaged and dead cells of the body. High $\mathrm{Ca}^{2+}$ concentration $\left(\geq 10^{-5} \mathrm{~mol} / \mathrm{l}\right)$ activates the enzyme Peptidyl Arginine Deiminase (PADI) and stimulates the conversion of arginine into citrulline. Normal level of $\mathrm{Ca}^{2+}$ inside the body is about 100 times lower. In damaged and dead cells, the cell membrane losses its selective permeability and allows excess entry of extracellular $\mathrm{Ca}^{2+}$ ions. Intracellular proteins like vimentin, nuclear histone become rapidly citrullinated and citrullination enhances cell-death process (van Venrooij et al., 2011).

But mere presence of citrullinated proteins does not stimulate the immune system to produce anti-CCP antibodies, and several genetic factors of the patient are thought to influence it strongly. Many studies have indicated the association between HLA-DRB1 Shared Epitope (SE) alleles and RA (Klareskog et al., 2009). Hill et al. (2009) demonstrated that the conversion of arginine to citrulline at the peptide side-chain position that interacts with the SE, significantly increases peptide-MHC affinity and leads to the activation of CD4+ T cells in DR4 IE transgenic mice.

Conversion of arginine into citrulline generates 'altered self' peptides that can be bound and presented by DRB1*1001, one of several SE alleles that is also strongly associated with RA and anti-CCP antibodies. Gyetvai et al. (2010) showed that, in particular, the S2 and S3P alleles (both associated with increased risk of RA) predisposed individuals to the production of antiCCP and anti-MCV (Mutated Citrullinated Vimentin) antibodies.

In June, 2010 the American College of Rheumatology (ACR) and the European League Against Rheumatism (EULAR) revised the 1987 ACR classification criteria for RA and introduced anti-CCP antibody estimation in an effort to improve early diagnosis of RA (Kasper et al., 2015). However in the early stages of the disease, it becomes difficult to make the diagnosis (Vallbracht and Helmke, 2005). Furthermore, in several studies conducted by Kroot et al. (2000), Quinn et al. (2006) and Kashyap et al. (2015) some variabilities in the results of anti-CCP positivity in rheumatoid arthritis patients have been observed.

Considering all these variability, present study was carried out to combine anti-CCP ELISA, RF IgM ELISA and RF latex agglutination tests to observe the combined sensitivity and specificity of the tests to diagnose rheumatoid arthritis more accurately.

\section{Materials and Methods}

\section{Study Design}

The study was carried out over a period of one year from August 2014 to July 2015. It was conducted on a total of 88 patients presenting with history of polyarthritis and for selection of cases and controls, they were subjected to detailed history, clinical examinations and necessary laboratory investigations. All the patients under study were divided into two groups of which the study group was composed of 57 patients with rheumatoid arthritis. The diagnosis was based on clinical features and "The New 2010 American College of Rheumatology (ACR) and the European League Against Rheumatism (EULAR) criteria" for the diagnosis of rheumatoid arthritis and on the expert opinion of attending physician of Rheumatology OPD. The control group was composed of 31 patients having non-RA rheumatic diseases with joint pain. The demographic profile of the patient such as age, sex, religion, residence, education and occupation was taken. The study group consisted of $42(73.68 \%)$ females and 15 $(26.32 \%)$ males with a ratio of $2.8: 1$, while the control group consisted of $12(38.71 \%)$ females and 19 $(61.29 \%)$ males with a ratio of $1: 1.6$. In the control group males were more than the females.

\section{Ethical Clearance}

The study proposal with relevant documents was submitted to the Institutional Ethics Committee (IEC) for review. The study commenced after receiving ethical approval and clearance certificate. Participation in the study was voluntary and a signed consent form was obtained from all the patients.

\section{Sample Collection}

About $5 \mathrm{ml}$ of venous blood was collected from each patient aseptically in a sterile vial and the blood was allowed to clot for separation of serum. Then in the Microbiology laboratory the serum was completely separated by centrifuging the sample vials in a centrifuge machine at 3000 revolutions per minute (rpm) for $5 \mathrm{~min}$. The separated serums were then transferred to sterile vials, labeled properly with serial numbers and stored at $-80^{\circ} \mathrm{C}$ till the assay was done.

Laboratory methods: All the sera of the patients were examined by anti-CCP ELISA Kit, (Omega Genesis, BioMerieux) for detection of anti-CCP IgG antibody. The results were expressed in Optical Density (OD) along yaxis and corresponding antibody titer of the six standards, plotted along x-axis of the curve. Subsequently the sera were examined for IgM Rheumatoid Factor by 
AutostatTMII Rheumatoid Factor IgM ELISA Kit (Hycor Biomedical, Garden Grove, California, USA). Rheumatoid Factor (RF) assay was also done by Latex Agglutination Slide Test with RHELAX-RF reagent Kit, (Tulip Diagnostics (P) Ltd. India).

\section{Statistical Analysis}

GraphPad inStat software and online Med Calc. statistical calculator were used to do statistical analyses of the test results. The distribution of laboratory test results were compared between study group and control group using the Fisher's exact test where necessary. Diagnostic utility of the tests were described by determining the sensitivity, specificity, Positive Predictive Value (PPV) and Negative Predictive Value (NPV) obtained with the cut-off value mentioned by the respective commercial kits. Assuming the prevalence of RA in the community as $0.5 \%$ corrected PPV and NPV was calculated using Bayes' theorem. Correlation between variables was assessed by Pearson correlation.

\section{Results}

Anti-CCP antibody was positive in 52(91.23\%) out of 57 cases in the study group and was negative in only $5(8.77 \%)$ out of 57 cases. The picture was different in case of control group, as anti-CCP antibody was positive in $9(29.03 \%)$ out of 31 cases in this group. Anti-CCP antibody was negative in 22 out of $31(70.97 \%)$ cases in the control (Table 1). When the serological profile in the study group and control group was compared it was found to be significant.

So according to the formula sensitivity, specificity, positive predictive value and negative predictive value of anti-CCP test is $91.23 \%, 70.97 \%, 75.77 \%$ and $89 \%$ respectively. Here, specificity of anti-CCP antibody test is showing lower value though the sensitivity of the test is high.

Rheumatoid Factor profiles as done by IgM ELISA was positive in $43(75.44 \%)$ and negative in $14(24.56 \%)$ out of a total of 57 cases in the study group (Table 2). Rheumatoid Factor (IgM ELISA) in cases of control group was positive in $10(32.26 \%)$ and negative in $21(67.74 \%)$ cases respectively. When the serological profile of Rheumatoid Factor IgM ELISA in the study group and control group was compared, it was found to be significant. $(p=0.0002)$.

After application of formula the sensitivity of Rheumatoid Factor IgM ELISA is $75.44 \%$ and specificity is $67.74 \%$. Positive Predictive Value (PPV) is $70.07 \%$ and Negative Predictive Value (NPV) of the test is $73.31 \%$.

Similarly the sensitivity of Rheumatoid Factor Latex agglutination test is $40.35 \%$ and specificity is $90.32 \%$. Positive Predictive Value (PPV) of the test is $80.65 \%$ and Negative Predictive Value (NPV) is $60.22 \%$.
Table 1. Serological profile in the study and control group

\begin{tabular}{lll}
\hline Test & Study Group & Control Group \\
\hline anti-CCP positive & $52(91.23 \%)$ & $9(29.03 \%)$ \\
& $($ True Positive) & (False Positive) \\
anti-CCP negative & $5(8.77 \%)$ & $22(70.97 \%)$ \\
& $($ False negative) & (True Negative) \\
Total $(\mathrm{n}=88)$ & 57 & 31 \\
& $\mathrm{p}<0.0001$ & \\
\hline
\end{tabular}

Table 2. Serological profiles of RF IgM ELISA in the study and control group

\begin{tabular}{lll}
\hline Test & Study Group & Control Group \\
\hline RF IgM positive & $43(75.44 \%)$ & $10(32.26 \%)$ \\
& (True Positive) & (False Positive) \\
RF IgM negative & $14(24.56 \%)$ & $21(67.74 \%)$ \\
& (False Negative) & (True Negative) \\
Total $(\mathrm{n}=88)$ & 57 & 31 \\
& $\mathrm{p}=0.0002$ & \\
\hline
\end{tabular}

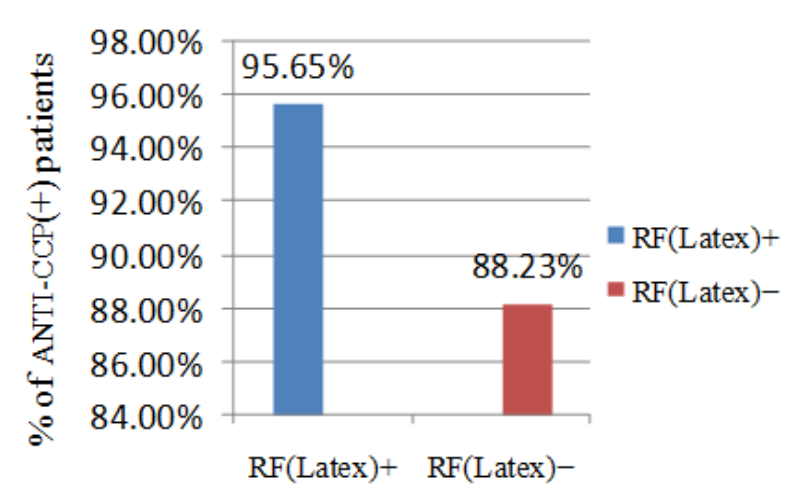

Fig. 1. Percentage of anti-CCP positive( $(+)$ patients in Latex test + and - patient

Here sensitivity of RF IgM ELISA (75.44\%) is higher than the sensitivity of RF Latex agglutination test $(40.35 \%)$ which is statistically extremely significant with $\mathrm{p}$ value of 0.0003 using Fisher's exact test. However specificity of RF IgM ELISA $(67.74 \%)$ is lower than the specificity of RF Latex agglutination test (90.32\%) which is statistically not quite significant $(p=0.0586)$.

The correlation coefficient of anti-CCP ELISA and rheumatoid factor Latex test is 0.3551 (95\% CI, 0.1573 to $\left.0.5255, \mathrm{r}^{2}=0.1261\right)$. The two tailed $\mathrm{p}$ value is 0.0007 which is highly significant. The same for anti-CCP ELISA and rheumatoid factor IgM ELISA is 0.6318 (95\% CI, 0.4867 to $\left.0.7430, \mathrm{r}^{2}=0.3992\right)$. The two tailed $\mathrm{p}$ value is $<0.0001$ which is extremely significant. The correlation coefficient for RF IgM ELISA and RF Latex test is 0.7679 (95\% CI 0.6655 to $\left.0.8420, \mathrm{r}^{2}=0.5897\right)$. The two tailed $\mathrm{p}$ value is $<0.0001$ which is extremely significant.

In this study, citrullinated peptide antibody test is positive in $95.65 \%$ RF Latex positive patients versus 88.23\% RF Latex negative patients (Fig.1). 


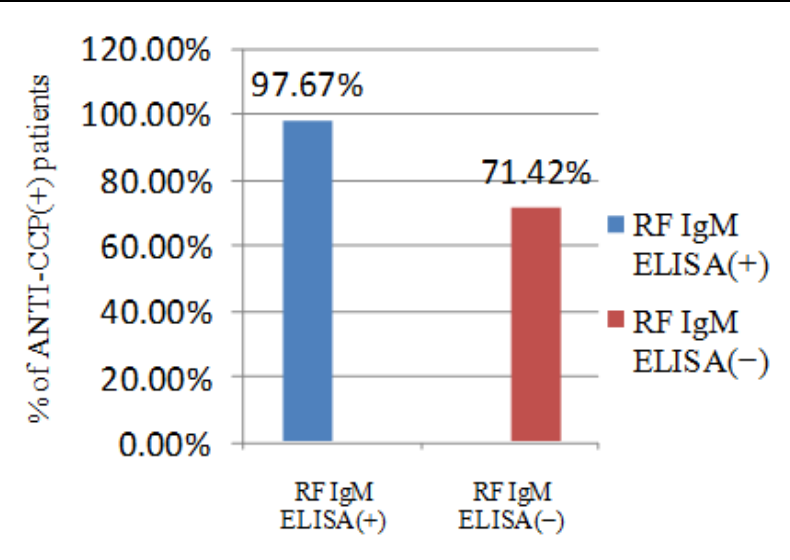

Fig. 2. Percentage of anti-CCP positive(+) patients in RF IgM ELISA + and - patients

Table 3. Sensitivity and specificity of different tests in the present study

\begin{tabular}{lll}
\hline Test & Sensitivity & Specificity \\
\hline Anti-CCP IgG ELISA & $91.23 \%$ & $70.97 \%$ \\
RF IgM ELISA & $75.44 \%$ & $67.74 \%$ \\
RF Latex test & $40.35 \%$ & $90.32 \%$ \\
Combined anti-CCP ELISA & $68.82 \%$ & $90.64 \%$ \\
+ RF IgM ELISA & & \\
Combined anti-CCP & $36.81 \%$ & $97.19 \%$ \\
ELISA + RF Latex test & & \\
\hline
\end{tabular}

Similarly anti-CCP antibody is positive in $97.67 \%$ RF IgM ELISA positive patients versus $71.42 \%$ RF IgM ELISA negative patients (Fig. 2). So we can say that anti-CCP antibody can be found more often in RF Latex test and RF IgM ELISA positive patients.

In normal clinical practice, to make a diagnosis more confidently, the results of two independent tests can be combined. To increase the specificity of a test, two tests can be combined by using the following formula:

Specificity of combined test $=1-$

$(1$-specificity of test 1$) \times(1$-specificity of test 2$)$

Here combining anti-CCP assay and Rheumatoid Factor Latex test, the combined specificity is increasing to $97.19 \%$ (Table 3) which indicates that, in a polyarthritis patient if both anti-CCP antibody and RF (Latex test) is negative then there is $97.19 \%$ chance that the patient is negative for rheumatoid arthritis.

But one drawback in combining these two tests in this way is that here the resultant sensitivity of the two tests is $36.81 \%$ which is less than the individual sensitivity of anti-CCP and RF (Latex test) assay.

Still if the specificity of the combined test is $97.19 \%$, we can safely say that in a polyarthritis patient if both anti-CCP and RF (Latex test) is negative then there is $97.19 \%$ chance that the patient is negative for rheumatoid arthritis.
Similarly if anti-CCP antibody test is combined with RF IgM ELISA then combined specificity of the two tests is $90.64 \%$ and the resultant sensitivity is $68.82 \%$ (Table 3).

After comparing all the results it is seen that combining these two results can more efficiently diagnose rheumatoid arthritis because there is $90-97 \%$ chance of the patient not having rheumatoid arthritis though the resultant sensitivity is reduced (Table 3).

Similarly by combining the anti-CCP ELISA, RF IgM ELISA and RF (Latex test), the combined specificity of the three tests can be calculated using the following formula:

Specificity of three combined tests

$=1-(1-$ specificity of test 1$) \times(1-$ specificity of test 2$)$ $\times(1-$ specificity of test 3$)$

Combined specificity of the three tests is $99.09 \%$ which means that if a patient is negative for all the three tests of anti-CCP ELISA, RF IgM ELISA and RF Latex test, then we may be $99.09 \%$ sure that the patient is not having rheumatoid arthritis.

\section{Discussion}

The present study was done to compare citrullinated peptide antibody and rheumatoid factor assay, individually and in combination to detect rheumatoid arthritis patients. Several studies have shown that citrullinated peptide antibody test is highly specific and could be detected early in the disease course and also predict the trends of progressive erosive disease. However, some variability of the results has also been found.

In the present study, sensitivity of Rheumatoid Factor IgM ELISA is $75.44 \%$ and specificity is $67.74 \%$. In a separate study conducted by Bas et al. sensitivity of Rheumatoid Factor IgM ELISA was $73 \%$ and specificity was $82 \%$ respectively (Bas et al., 2003). In the present study, sensitivity of Rheumatoid Factor (Latex agglutination test) is $40.3 \%$ and specificity is $90.32 \%$ respectively. In another study conducted by Saraux et al. (2003) sensitivity of RF Latex test was $45 \%$. In a different study Aflaky et al. (2010) reported similar values of RF Latex test. Nishimura et al. (2007) reported that sensitivity of RF is $69 \%$ and specificity is $85 \%$.

In the present study, sensitivity of RF IgM ELISA (75.44\%) is better than the sensitivity of RF Latex test $(40.3 \%)$ which is statistically extremely significant $(\mathrm{p}=$ 0.0003). Swedler et al. (1997) has also mentioned that sensitivity of RF IgM ELISA is better than the sensitivity of RF Latex test. Niewold et al. (2007) has mentioned that in several studies it has been observed that rheumatoid factor showed a variable sensitivity of $31 \%$ to $54 \%$ and specificity of $91 \%$ to $93 \%$ for the eventual diagnosis of RA. 
In the current study sensitivity of anti-CCP antibody is $91.23 \%$ and specificity is $70.97 \%$ respectively. In another study conducted by Gupta et al. (2009) sensitivity and specificity of anti-CCP antibody was 85 and $90.19 \%$ respectively. In a study conducted by Schellekens et al. (2000), sensitivity and specificity of anti-CCP antibody was 68 and $98 \%$ respectively. Goldbach-Mansk et al. (2000) reported that sensitivity and specificity of anti-CCP antibody was $50 \%$ and $90 \%$ respectively. According to Bizzaro et al. (2001) sensitivity and specificity of anti-CCP antibody was $41 \%$ and $98 \%$ respectively. In a study Bas et al. (2002) showed that sensitivity and specificity of anti-CCP antibody was $68 \%$ and $96 \%$ respectively. Suzuki et al. (2003) reported that sensitivity and specificity of antiCCP antibody was $88 \%$ and $89 \%$ respectively. So it is quite obvious that in the present study, citrullinated peptide antibody test is showing reduced specificity in comparison to the other studies.

There may be several explanations for the difference between reported sensitivity and specificity of anti-CCP antibody test in different studies. One explanation for the discrepancy is that the differences in the patient populations (mainly disease duration) among these studies might have some influence on the results. Another study showed that the specificity and sensitivity of anti-CCP antibodies may depend on the patient's race (Binesh et al., 2014). It is also probably inevitable that sensitivity and specificity of laboratory tests will vary between studies as there will be local variations in the application of clinical diagnostic criteria.

To overcome discrepancies of individual test results, if anti-CCP ELISA, RF IgM ELISA and RF Latex test are combined, then the combined specificity of the three tests is $99.09 \%$ which is quite helpful in establishing diagnosis. Parikh et al. has also shown that to make diagnosis of a disease more accurately three diagnostic tests can be combined (Parikh et al., 2008).

\section{Conclusion}

Though citrullinated peptide antibody assay can help significantly in diagnosis of RA, all the present study can realistically do is provide a suggestion to clinicians that combining multiple blood test results like anti-CCP antibody, RF IgM assay and RF Latex agglutination tests may provide an output that more strongly predicts clinical diagnosis.

\section{Acknowledgement}

All the staffs of the Department of Microbiology and the Department of Medicine of Gauhati Medical College and Hospital, Guwahati, where this study was carried out.

\section{Author's Contributions}

Anindya Das: Performed the experiments, prepared data analyses report and wrote the manuscript.

Chimanjita Phukan: Assisted in revising, data analyses and improving the paper.

Chitralekha Baruah: Assisted in collecting patients' history and data from Rheumatology Out Patient Department.

\section{Ethics}

The article is original in all respect. All the authors have participated and approved the manuscript and no ethical issues are related to it.

\section{References}

Arnett, F.C., S.M. Edwowrty, D.A. Bloch, D.J. Mcshane and J.F. Fries et al., 1988. The American Rheumatism Association 1987 revised criteria for the classification of Rheumatoid Arthritis. Arthritis Rheum, 31: 315-324. DOI: 10.1002/art.1780310302

Aflaky, E., S. Shenavandeh and M.J. Ashraf, 2010. A comparison of performance of anti-cyclic citrullinated peptide 2 and citrullinated protein antibodies in the diagnosis of rheumatoid arthritis in Iranian patients. Rheumatol Int., 30: 461-466. DOI: $10.1007 / \mathrm{s} 00296-009-0980-\mathrm{x}$

Bas, S., S. Genevay, O. Meyer and C. Gabay, 2003. Anti-cyclic citrullinated peptide antibodies, IgM and $\operatorname{IgA}$ rheumatoid factors in the diagnosis and prognosis of rheumatoid arthritis. Rheumatology, 5: 677-680. DOI: 10.1093/rheumatology/keg184

Binesh, F., H.S. Salehabadi, N. Behniafard, K. Ranginkaman and N. Behniafard, 2014. A comparative assessment of the diagnostic value of anti-cyclic citrullinated peptide antibodies and rheumatoid factor in rheumatoid arthritis. J. Clin. Exp. Pathol., 4: 158. DOI: $10.4172 / 2161-0681.1000158$

Bizzaro, N., G. Mazzanti, E. Tonutti, D. Villalta and R. Tozzoli, 2001. Diagnostic accuracy of the anticitrulline antibody assay for rheumatoid arthritis. Clin. Chem., 47: 1089-1093.

Brian, J.S., A.L. Corper, M.K. Sohi, R. Jefferis and D. Beale et al., 1998. The structure of a human rheumatoid factor bound to $\mathrm{IgG} \mathrm{Fc}$. Glycoimmunology 2 Advances in Experimental Medicine and Biology 1998; 435: 41-50. DOI: 10.1007/978-1-4615-5383-0_4

Goldbach-Mansky, R., J. Lee, A. McCoy, J. Hoxworth and C. Yarboro et al., 2000. Rheumatoid arthritis associated autoantibodies in patients with synovitis of recent onset. Arthritis Res., 2: 236-243.

DOI: $10.1186 / \mathrm{ar} 93$ 
Guermazi, A., B. Taouli, J.A. Lynch and C.G. Peterfy, 2004. Imaging of bone erosion in rheumatoid arthritis. Semin Musculoskelet Radiol., 4: 269-85. DOI: $10.1055 / \mathrm{s}-2004-861575$

Gupta, R., M.M. Thabah, R. Aneja and P.J. Chandrasenan, 2009. Usefulness of Anti-CCP antibodies in rheumatic diseases in Indian patients. Indian J. Med. Sci., 3: 92.

DOI: $10.4103 / 0019-5359.49285$

Gyetvai, Á., Z. Szekanecz, L. Soós, Z. Szabó and A. Fekete et al., 2010. New classification of the shared epitope in rheumatoid arthritis: Impact on the production of various anti-citrullinated protein antibodies. Rheumatology, 49: 25-33.

DOI: 10.1093/rheumatology/kep338

Hill, J.A., S. Southwood, A. Sette, A.M. Jevnikar and D.A. Bell et al., 2003. Cutting edge: The conversion of arginine to citrulline allows for a high-affinity peptide interaction with the rheumatoid arthritisassociated HLA-DRB1*0401 MHC class II molecule. J. Immunol., 171: 538-541. DOI: $10.4049 /$ jimmunol.171.2.538

Kashyap, B., U. Tiwari, A. Garg and I.R. Kaur, 2015. Diagnostic utility of anti-CCP antibodies and rheumatoid factor as inflammatory biomarkers in comparison with C-reactive protein and TNF- $\alpha$ in rheumatoid arthritis. Trop J. Med. Res., 18: 5-9. DOI: $10.4103 / 1119-0388.152534$

Kasper, D.L., A.S. Fauci, S.L. Hauser, D.L. Longo and J.L. Jameson et al., 2015. Harrison's Principles of Internal Medicine 19/E (Vol.1 \& Vol.2). 19th Edn., McGraw Hill Professional, New York, ISBN10: 0071802169 , pp: 3000.

Klareskog, L., A.I. Catrina and S. Paget, 2009. Rheumatoid arthritis. Lancet, 373: 659-672. DOI: $10.1016 / \mathrm{S} 0140-6736(09) 60008-8$

Kroot, E.J., B.A.W. de Jong, M.A. van Leeuwen, H. Swinkels and F.H.J. van den Hoogen et al., 2000. The prognostic value of anti-cyclic citrullinated peptide antibody in patients with recent-onset rheumatoid arthritis. Arthritis Rheum., 43: 1831-1835.

DOI: $10.1002 / 1529-0131(200008) 43: 8<1831:: A I D-$ ANR19>3.0.CO;2-6

Lee, D.M. and P.H. Schur, 2003. Clinical utility of the anti-CCP assay in patients with rheumatic disease. Ann. Rheum Dis., 62: 870-874. DOI: $10.1136 /$ ard.62.9.870

Nienhuis, R.L. and E. Mandema, 1964. A new serum factor in patients with rheumatoid arthritis: The antiperinuclear factor. Ann. Rheum. Dis., 23: 302-305. DOI: 10.1136/ard.23.4.302

Niewold, T.B., M.J. Harrison and S.A. Paget, 2007. Anti-CCP antibody testing as a diagnostic and prognostic tool in rheumatoid arthritis. Q. J. Med., 100: 193-201. DOI: 10.1093/qjmed/hcm015
Nishimura, K., D. Sugiyama, Y. Kogata, G. Tsuji and T. Nakazawa et al., 2007. Metaanalysis: Diagnostic accuracy of anti-cyclic citrullinated peptide antibody and rheumatoid factor for rheumatoid arthritis. Ann. Internal Med., 146: 797-808. DOI: /10.7326/00034819-146-11-200706050-00008/10.7326/00034819-146-11-200706050-00008

O'Dell, J.R., 2003. Treating rheumatoid arthritis early: A window of opportunity? Arthritis Rheum., 46: 283-285. DOI: 10.1002/art.10092

Parikh, R., A. Mathai, S. Parikh, G.C. Sekhar and R. Thomas, 2008. Understanding and using sensitivity, specificity and predictive values Indian. J. Ophthalmol., 1: 45-50.

DOI: $10.4103 / 0301-4738.37595$

Quinn, M.A., A.K. Gough, M.J. Green, J. Devlin and E.M. Hensor et al., 2006. Anti-CCP antibodies measured at disease onset help identify seronegative rheumatoid arthritis and predict radiological and functional outcome. Rheumatology, 45: 478-80. DOI: 10.1093/rheumatology/kei203

Saraux, A., J.M. Berthelot, V. Devauchelle, B. Bendaoud and G. Chalès et al., 2003. Value of antibodies to citrulline-containing peptides for diagnosing early rheumatoid arthritis. J. Rheumatol., 30: 2535-9. PMID: 14719190

Schellekens, G.A., B.A.W. de Jong, F.H.J. van den Hoogen and L.B.A. van de Putte, 1998. Citrulline is an essential constituent of antigenic determinants recognized by rheumatoid arthritis-specific autoantibodies. J. Clin. Investig., 101: 273-281. DOI: $10.1172 / \mathrm{JCI} 1316$

Schellekens, G.A., H. Visser, B.A. de Jong, F.H. van den Hoogen and J.M. Hazes et al., 2000. The diagnostic properties of rheumatoid arthritis antibodies recognizing a cyclic citrullinated peptide. Arthritis Rheum, 43: 155-63. DOI: 10.1002/15290131(200001)43:1<155::AID-ANR20>3.0.CO;2-3

Sebbag, M., M. Simon, C. Vincent, C. Masson-Bessiere and E. Girbal et al., 1995. The antiperinuclear factor and the socalled antikeratin antibodies are the same rheumatoid arthritisspecific autoantibodies. J. Clin. Investig., 95: 2672-2679. DOI: 10.1172/JCI1 17969

Soltys, A.J. and J.S. Axford, 1997. Rheumatoid factors: Where are we now? Ann. Rheum Dis., 56: 285-286. DOI: 10.1136/ard.56.5.285

Suzuki, K., T. Sawada, A. Murakami, T. Matsui and S. Tohma et al., 2003. High diagnostic performance of ELISA detection of antibodies to citrullinated antigens in rheumatoid arthritis. Scand J. Rheumatol., 32: 197-204. DOI: $10.1080 / 03009740310003677$ 
Swedler, W., J. Wallman, C.J. Froelich and M. Teodorescu, 1997. Routine measurement of IgM, $\mathrm{IgG}$ and IgA rheumatoid factors: High sensitivity, specificity and predictive value for rheumatoid arthritis. J. Rheumatol., 6: 1037-44. PMID: 9195506

Vallbracht, I. and K. Helmke, 2005. Additional diagnostic and clinical value of anti-cyclic citrullinated peptide antibodies compared with rheumatoid factor isotypes in rheumatoid arthritis. Autoimmun Rev., 4: 389-94.

DOI: 10.1016/j.autrev.2005.02.001 van Venrooij, W.J., J.J.B.C. van Beers and G.J. M. Pruijn, 2011. Anti-CCP antibodies: The past, the present and the future. Nat. Rev. Rheumatol., 7: 391-398. DOI: 10.1038/nrrheum.2011.76

Young, B.J., 1979. Anti-keratin antibodies in rheumatoid arthritis. Br. Med. J., 2: 97-9.

DOI: $10.1136 / \mathrm{bmj} .2 .4670 .97$

Waaler, E., 1940. On the occurrence of a factor in human serum activating the specific agglutination of sheep red corpuscles. Acta Pathol Microbiol Scand, 17: 172-88. 